\title{
La satisfacción docente y práctica pedagógica durante la emergencia sanitaria
}

\author{
Teacher satisfaction and pedagogical practice during the health
}

Satisfação do professor e prática pedagógica durante emergências de saúde

\section{ARTíCULO DE INVESTIGACIÓN}

Ana María Seje Jallorana

anamarsj@hotmail.com

https://orcid.org/0000-0002-3539-4842

Universidad César Vallejo, Lima-Perú

Madeleine Rivera Cuzco

madeleine_rivera@hotmail.com

https://orcid.org/0000-0002-9633-8529

Universidad César Vallejo, Callao-Perú
Aracelli Gonzales-Sánchez

dgonzalessa@ucvvirtual.edu.pe

https://orcid.org/0000-0003-0028-9177

Universidad César Vallejo, Lima-Perú

CIFE, Cuernavaca-México

\section{Luis Huaringa Tello}

lhuaringa@ucv.edu.pe

https://orcid.org/0000-0002-6582-7776

Universidad César Vallejo, Callao-Perú

Recibido 21 de mayo 2021 | Arbitrado y aceptado 15 de junio 2021 | Publicado en 01 julio 2021

\section{RESUMEN}

La emergencia sanitaria determinó en el año 2020, se implemente la educación remota o a distancia, de tal manera los docentes tuvieron que asumir nuevas estrategias para desempeñar su práctica pedagógica. El objetivo de este estudio fue determinar la incidencia de la satisfacción docente en su práctica pedagógica durante el contexto de la emergencia sanitaria. La metodología empleada fue de enfoque cuantitativo con diseño descriptivo, se empleó como instrumento una encuesta en escala de Likert, que se aplicó a 112 docentes pertenecientes a colegios del Cono Norte de Lima. Los resultados obtenidos permitieron concluir que la percepción de los docentes evidenció que existe incidencia entre la satisfacción docente y su práctica pedagógica en el nivel competente.

Palabras clave: Docente; Emergencia; Práctica; Pedagógica; Sanitaria; satisfacción
ABSTRACT

RESUMO
The health emergency determined in 2020, remote or distance education was implemented, in such a way that teachers had to assume new strategies to carry out their pedagogical practice. The objective of this study was to determine the incidence of teacher satisfaction in their pedagogical practice during the context of the health emergency. The methodology used was a quantitative approach with a descriptive design, a Likert scale survey was used as an instrument, which was applied to 112 teachers belonging to schools in the Northern Cone of Lima. The results obtained allowed us to conclude that the teachers' perception showed that there is an incidence between teacher satisfaction and their pedagogical practice at the competent level.

Key words: Teacher; emergency; practice; pedagogical; health; satisfaction
Implantou-se a emergência sanitária determinada em 2020, a educação a distância ou a distância, de tal forma que os professores tiveram que assumir novas estratégias para realizar sua prática pedagógica. $\mathrm{O}$ objetivo deste estudo foi determinar a incidência da satisfação do professor em sua prática pedagógica durante o contexto da emergência sanitária. A metodologia utilizada foi uma abordagem quantitativa com desenho descritivo, utilizou-se como instrumento um survey em escala Likert, o qual foi aplicado a 112 professores pertencentes a escolas do Cone Norte de Lima. Os resultados obtidos permitiram concluir que a percepção dos professores mostrou que existe uma incidência entre a satisfação do professor e a sua prática pedagógica no nível competente.

Palavras-chave:

Professor;

Emergência; Prática; Pedagógica; Saúde, Satisfação 


\section{INTRODUCCIÓN}

El establecimiento del confinamiento obligatorio en el Perú, debido a la emergencia sanitaria producto del COVID-19, ha determinado importantes cambios en el desarrollo regular de las actividades, entre ellas la educación, que pasó de ser presencial a distancia o remota, los hogares se han convertido en escuelas (Gómez y Escobar 2021) este nuevo escenario exigió a los miembros de la comunidad educativa, adecuarse a este nuevo proceso de como brindar las experiencias de aprendizaje; por consiguiente, fue necesario explorar como se llevaría la práctica profesional, además explorar en ellos el tema de la satisfacción docente y su práctica pedagógica.

La satisfacción docentes desde la satisfacción laboral, manifestada por López y Gallegos (2014) de acuerdo a los principales teóricos sobre el tema de Locke y Latham, la satisfacción laboral se concibió como un buen estado emocional positivo de agrado, en el cual el trabajador se siente valorado en su centro laboral; en esta línea a mejor satisfacción mayor productividad; para (Sánchez y García, 2017) involucra la actitud o actitudes que tiene el individuo con su centro laboral. En tal sentido en una organización o institución, el estado anímico del trabajador será fundamental para su desempeño laboral.

Para Cantón y Tellez (2016) "sobre la satisfacción profesional del docente, tratan de comprender si los determinantes de la satisfacción residen en la naturaleza del trabajo en sí, en las variables inherentes al profesional o si es resultado de las interacciones establecidas entre el profesional y las especificidades de su contexto de trabajo, como las interacciones sociales, el clima, el introducir en la enseñanza nuevos recursos y metodologías ( $p$. 280). Por consiguiente, en este estudio consideró la satisfacción docente como el estado anímico en el cual los profesores desempeñan su práctica profesional, es importante precisar que, también se consideran dos etapas: la primera será antes de la emergencia sanitaria y la segunda después del estallido de la emergencia sanitaria.

La variable de estudio satisfacción docente, fue dimensionada de la siguiente manera: la realización profesional, la cual comprende el adecuado desarrollo de la profesión, en el Perú existe la Carrera Pública Magisterial, es importante la capacitación constante y permanente, además se toma en cuenta la meritocracia en función a evaluaciones de acuerdo al desempeño docente, Ventura Montes (2020) el ser parte de la Carrera Pública involucra también investigar, esto contribuirá a brindar una educación de calidad. Poma (2014) señaló que, en América Latina la meritocracia es parte de las políticas públicas para evaluar el desempeño profesional docente. El estallido de la emergencia sanitaria, exigió que los docentes de manera acelerada sean insertados en el contexto de la enseñanza a distancia o remota, de la cual no estaban adecuadamente preparados. (Picón et al., 2020) estudiaron como en Paraguay con el objetivo de mantener la continuidad académica, los docentes tuvieron que asumir este reto, afrontando dificultades tan iguales que sus estudiantes, de adaptarse a este nuevo escenario y las dificultades de acceso a la tecnología. Situación similar afrontó el Perú, dese el Ministerio de Educación (Minedu), se implementaron capacitaciones aceleradas para mejorar su desempeño profesional haciendo uso de la Plataforma Aprendo en Casa y PerúEduca.

La Defensoria del Pueblo (2020) brindó un análisis de la situación de la educación durante la pandemia del COVID-19, del cual un dato interesante es que para el año 2017, "el 72,9\% de los profesores públicos manifestó que no recibieron capacitación en el uso de las TIC durante el año 2017. Además, el $38,7 \%$ de docentes manifestó que le gustaría recibir formación para fortalecer su desempeño en competencias digitales y uso de las TIC" (Defensoria del Pueblo, 2020, p. 23), de 
acuerdo a estas cifras, era evidente las dificultades que afrontaban los docentes antes de la pandemia en el uso de recursos digitales; en tal sentido adaptarse a este nuevo escenario resultó una situación muy complicada.

La siguiente dimensión que se consideró fue las relaciones interpersonales, $\tan$ necesarias para una adecuada convivencia, el reconocimiento, un adecuado trato entre todos los actores de la comunidad educativa, fortalecen la identificación con su institución y por consiguiente la satisfacción laboral, en el contexto de la emergencia sanitaria, se emitieron disposiciones por parte del Minedu, en el cual se involucró a las familia, para que contribuyan en el desarrollo de las experticias de aprendizajes de sus hijos, RV N 093-2020 (Minedu, 2020a), en tal sentido las familias serán los aliados de los docentes para el desarrollo de su práctica profesional, sin embargo en la línea de la convivencia para fortalecer las relaciones personales, se debieron establecer pautas de netiquetas, (Gordón, 2020) señaló que, la manera como se desarrolla las experiencias de aprendizaje en tiempos de pandemia es un ensayo y error, ya la educación no será la misma habrá que reformular el proceso de enseñanza aprendizaje cuando los estudiantes regresen a la educación presencial.

La tercera dimensión es la estructura organizativa, la satisfacción docente esta en relación, a cada una de los miembros de la comunidad educativa desde la gestión: directiva, administrativa, pedagógica y comunitaria. La cuarta dimensión que se consideró fue el ambiente físico; en la primera etapa antes de la emergencia sanitaria, era la escuela; sin embargo, el estallido de la educación remota involucró el teletrabajo, en tal sentido la manera de conectividad sea sincrónica o asincrónica, para Dos Santos et al., (2021) investigaron las afecciones emocionales de estrés que genera el teletrabajo en los docentes, siendo uno de los factores que las jornada laboral se altera el respeto de los tiempos y la excesiva carga laboral.

Para Chaves Torres, (2017) tomando lo aportes de Fierro (1990) la práctica pedagógica es una praxis social e intencionada, los maestros tanto en estaciones educativas públicas y privadas, su desempeño profesional adecuado tendrá como logró que los estudiantes desarrollen sus competencias, evidenciándose en el desarrollo de sus experiencias de aprendizaje, lamentablemente como se ha señalado la emergencia sanitaria, exigió que los docentes tengan que adecuarse a una nueva realidad para desarrollar su práctica profesional, esta situación develó debilidades, como fue el desarrollo de competencias digitales, OcañaFernández et al., (2020) los docentes deben estar preparados en el empleo de la tecnología y hacerla parte de sus sesiones de aprendizaje, Pozos Pérez y Tejada Fernández, (2018) el docente debe adecuarse a los cambios y estar atento a ellos.

De acuerdo a Zambrano (2018) "las prácticas pedagógicas como aquellas estrategias, instrumentos y acciones que el docente realiza en el aula para guiar el proceso de enseñanza aprendizaje, con la pretensión de desarrollar en el educando diversas competencias" (p. 70). En la práctica profesional docente asumen situaciones retadoras Castro et al., (2020) los llaman docentes de Inmunología, debido a que su labor profesional se realiza en tiempos de COVID-19, en tal sentido fueron competentes y capaces de sacar provecho a estas coyuntura para mejorar sus clases con nuevos recursos, en tal sentido Ceballos et al., (2021) dejaran una huella en el trabajo pedagógico de cómo se desarrollará las experiencias de aprendizaje, 
En función a lo expuesto, el objetivo de este estudio es determinar la incidencia de la satisfacción docente en su práctica pedagógica durante el contexto de la emergencia sanitaria. Así mismo; se considera importante porque es desarrollado dentro de la nueva realidad académica, esto permite a la comunidad académica obtener información del desempeño docente desde los propios actores.

\section{MÉTODO}

Se desarrolló una investigación que estuvo enmarcada en el paradigma positivista, con enfoque cuantitativa, de diseño descriptivo, se eligió como muestra de estudio a 112 docentes pertenecientes a instituciones públicas del Cono Norte de Lima, adscritos a la Unidad de Gestión Educativa (UGEL 02). Para medir la variable satisfacción docente, se construyó un instrumento en escala de Likert con 17 ítems, el cual fue aprobado para su aplicación mediante juicio de expertos, se determinó la confiabilidad, empleando una muestra de 43 docentes, obtuvo un Alfa de Cronbach de 0,951; y la fiabilidad de acuerdo a la prueba de Medida Kaiser
Meyer Olkin o índice (KMO) brindó un coeficiente de 0,813 , de tal manera que validó la aplicación del instrumento. Debido a las condiciones de la emergencia sanitaria, se solicitó los respectivos premisos a los directivos de las instituciones donde se realizó, además el instrumento se compartió entre los maestros mediante formulario de Google Forms, se distribuyó por correo electrónico y WhatsApp.

\section{RESULTADOS Y DISCUSIÓN}

De acuerdo al procesamiento estadístico, brindó los siguientes resultados en la estadística descriptiva.

La Tabla 1 consideró que para medir la satisfacción docente de acurdo a los niveles de Destacado, competente y básico, el resultado evidencia que de manera general la satisfacción docente se ubica en el nivel básico con 67,9\%; sin embargo, la interpretación se realiza por dimensiones, en el nivel básico se ubican se encontraron la realización personal y el ambiente físico. Además, se encontró una mejor percepción en el nivel competente las dimensione relaciones interpersonales y estructura organizacional.

Tabla 1. Niveles frecuencia les de satisfacción docente en estaciones educativas del Cono Norte de Lima.

\begin{tabular}{cccccc}
\hline Niveles & $\begin{array}{c}\text { Satisfacción } \\
\text { docente }\end{array}$ & $\begin{array}{c}\text { Realización } \\
\text { profesional }\end{array}$ & $\begin{array}{c}\text { Relaciones } \\
\text { interpersonales }\end{array}$ & $\begin{array}{c}\text { Estructura } \\
\text { organizativa }\end{array}$ & Ambiente físico \\
\hline po\% & $\mathbf{p o \%}$ & $\mathbf{p o \%}$ & $\mathbf{p o \%}$ & $\mathbf{p o \%}$ \\
\hline Destacado & 0.9 & 1.8 & 4.5 & 2.7 & 4.5 \\
Competente & 31.3 & 30.4 & 58.9 & 50.0 & 36.6 \\
Básico & 67.9 & 67.9 & 36.6 & 47.3 & 58.9 \\
Total & 100.0 & 100.0 & 100.0 & 100.0 & 100.0 \\
\hline
\end{tabular}




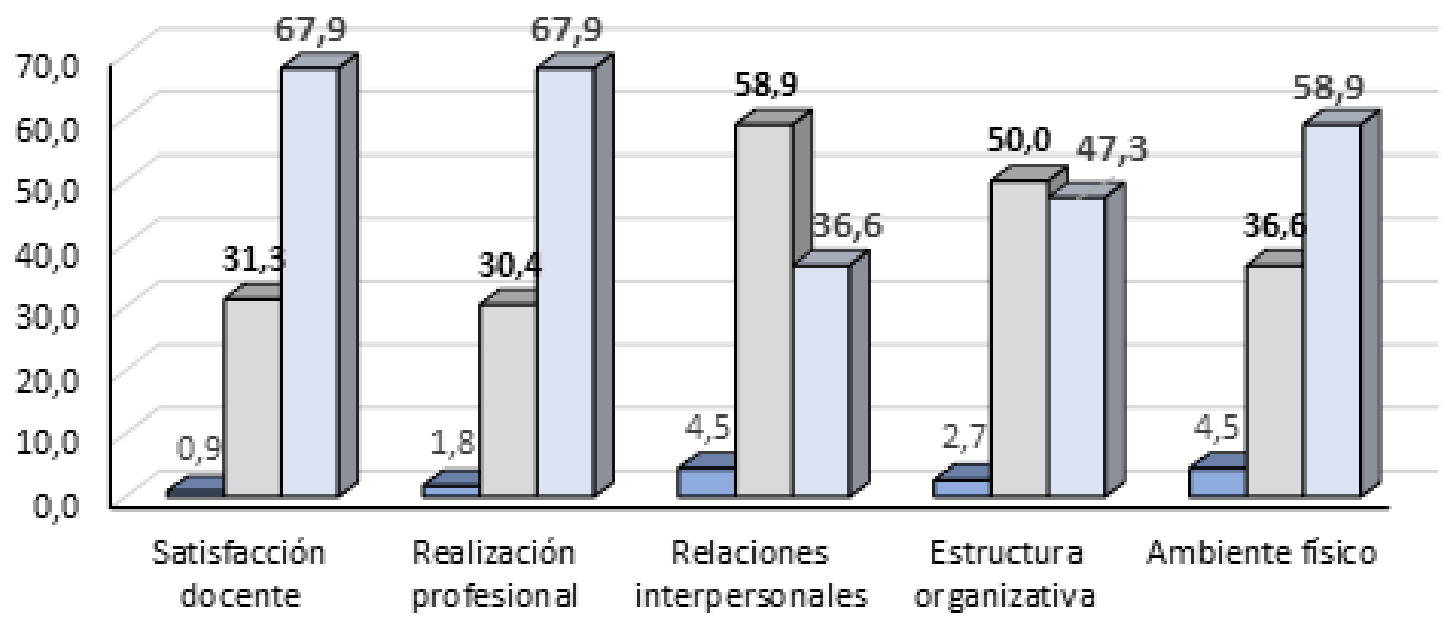

Figura 1. Niveles frecuencia les de satisfacción docente en estaciones educativas del Cono Norte de Lima.

De acuerdo a la información de la Figura 1 se pudo inferir en los gráficos que las dimensiones relaciones interpersonales y ambiente físico han obtenido el mejor resultado, ambas coincidieron con el 36,6\%.

\section{Resultados inferenciales}

Para la interpretación de los resultados, se empleó estadístico de prueba: Regresión logística y el coeficiente de prueba Nagelkerke.

Tabla 2. Coeficientes de la satisfacción docente en la práctica pedagógica en instituciones educativas del Cono Norte de Lima.

\section{Estimaciones de parámetro}

\begin{tabular}{|c|c|c|c|c|c|c|c|}
\hline & & \multirow{2}{*}{ Estimación } & \multirow{2}{*}{ Desv. Error } & \multirow{2}{*}{ Wald } & \multirow{2}{*}{ Sig. } & \multicolumn{2}{|c|}{$\begin{array}{l}\text { Intervalo de confianza } \\
\text { al } 95 \%\end{array}$} \\
\hline & & & & & & $\begin{array}{l}\text { Límite } \\
\text { inferior }\end{array}$ & $\begin{array}{l}\text { Límite } \\
\text { superior }\end{array}$ \\
\hline \multirow[t]{5}{*}{ Umbral } & {$[\mathrm{prac}=\mathrm{bas}]$} & -24.485 & 1.159 & 446.162 & 0.000 & -26.757 & -22.213 \\
\hline & {$[\mathrm{prac}=\mathrm{com}]$} & -19.922 & 0.474 & 145.230 & 0.000 & -20.851 & -18.993 \\
\hline & & & & & & & -17.816 \\
\hline & [sat=bas.] & -4.387 & 2.657 & 2.727 & 0.020 & -9.594 & 0.820 \\
\hline & {$[\mathrm{sat}=\mathrm{com}]$} & -2.997 & 0.605 & 24.541 & 0.000 & -4.183 & -1.811 \\
\hline Ubicación & \multicolumn{2}{|c|}{ Cox y Snell 0.400} & \multicolumn{2}{|c|}{ Nagelkerke 0.563} & \multicolumn{2}{|c|}{ Nagelkerke 0.412} & \\
\hline
\end{tabular}

Función de enlace: Logit. 
Los resultados que se muestran en la Tabla 2, representan la incidencia de la práctica pedagógica y la satisfacción docente, mediante la regresión logística ordinal por las características de las escalas de las variables. La percepción de los docentes encuestados brindó como información en esta dimensión un valor en el coeficiente de Wald fue $24.541 \%$, se ubicó en el nivel competente, además fue significativo porque se obtuvo el valor de 0.000 en que p_valor $<0.05$ y un límite inferior de -1.811 .

\section{Discusión}

Para Moreno (2017) quien realizó una investigación cualitativa sobre la práctica pedagógica, entre las conclusiones explicó que, los docentes de manera inconsciente demuestran resistencia al cambio, en tal sentido las dificultades de los maestros van en la línea con el porcentaje obtenido de $67.9 \%$, el cual se ubicó la percepción de los docentes en un nivel básico en la dimensión realización profesional, se infirió que esto se debió a la coyuntura de la educación remota, en la cual de manera acelerada adquirieron competencias digitales para mejorar su práctica profesional.

Por otro lado, Torres (2015) investigó en Puno la incidencia de la gestión directiva con la práctica docente, lo realizó con 158 profesores, se encuentra en la línea de los hallazgos de este estudio, porque la emergencia sanitaria ha exigido que los directivos realicen acciones e intervenciones con los miembros de su comunidad educativa, para adecuar las experiencias de aprendizajes al nuevo contexto de la educación remota Gonzales-Sánchez et al., (2021) plantearon que los directores son los responsables de monitorear y acompañar a los docentes para el mejor desarrollo de su práctica profesional desde la gestión pedagógica. De acuerdo a los resultados que se obtuvo las dimensiones relaciones interpersonales $58,9 \%$ y estructura organizativa $50,0 \%$ se ubicaron en el nivel competente, lo que permitió inferir que en cierta mediad los profesores se sienten satisfechos con respecto a su desempeño profesional.

De acuerdo a los hallazgos de Díaz y Almagiáb (2017) realizaron un estudio de la resiliencia y la satisfacción laboral docente en 119 profesionales de escuelas públicas chilenas, los autores llegaron a concluir que a más edad del trabajador mejorar la resiliencia, en el estudio que se realizó no es considerada la edad, sin embargo de la encuesta aplicada a pesar de ser la emergencia sanitaria un contexto complicado, los docentes evidenciaron como adecuada, se infiere que de sentir descontento las repuestas se debieron ubicar en el nivel básico, en su totalidad, lo que no sucedió en esta investigación.

Rodríguez-Sosa y Hernández-Sánchez (2018) concluyeron que en la práctica pedagógica es impotente la toma de decisión de los docentes, de acuerdo al contexto que se encuentran, emplearon el termino priorizar; en el contexto de la emergencia sanitaria es importante precisar que se emitieron disposiciones para brindar orientaciones del desempeño y los aprendizajes priorizados en el periodo lectivo 2020, de acuerdo a la RV N 093 "Orientaciones pedagógicas para el servicio educativo de Educación Básica durante el año 2020 en el marco de la emergencia sanitaria por el Coronavirus COVID-19” (Minedu, 2020b), en función al estudio realizado coincide con la capacidad de adaptación de los docentes. 


\section{CONCLUSIONES}

Se concluye que existe incidencia entre la satisfacción docente y su práctica pedagógica de manera competente, esta información evidenció ser adecuada, realmente al implementar el estudio se creyó obtener datos negativos o de rechazo de los docentes; por esta razón se infirió que los docentes se adecuaron al nuevo escenario, a pesar de las dificultades que pudieron tener, como es el manejo de competencias digitales, las cuales fueron un obstáculo para que ellos diseñen sus sesiones de aprendizaje; así mismo, poder tener una apropiada conectividad con sus estudiantes.

De acuerdo la naturaleza de la muestra de estudio, se debe precisar que fueron docentes de instituciones públicas, las cuales no necesariamente por ser la emergida sanitaria evidenciaban dificultades, sino ya se detectaban con anterioridad de acuerdo a lo señalado por la Defensoria del Pueblo (2020) sobre la existencia de brechas digitales, determinando la poca conexión para lleva sus experiencias de aprendizaje en la plataforma Aprendo en Casa.

Finalmente, la emergencia sanitaria producto delCOVID-19 ha permitido explorar las dificultades que afrontan los docentes en su práctica profesional, sin embargo, siendo optimistas, ha significado una oportunidad para que los docentes fortalezcan sus competencias digitales y recursos a emplear en sus experiencias de aprendizajes.

\section{REFERENCIAS}

Cantón Mayo, I., y Téllez Martínez, S. (2016). La satisfacción laboral y profesional de los profesores. Revista Lasallista de Investigación, 13(1), 214-226. https://doi.org/10.22507/rli. v13n1a18
Castro, M., Paz, M., y Cela, E. (2020). Aprendiendo a enseñar en tiempos de pandemia COVID-19: nuestra experiencia en una universidad pública de Argentina. Revista Digital de Investigación En Docencia Universitaria, 14(2). https://doi. org/10.19083/ridu.2020.1271

Ceballos, E.Cedeño, E., Sánchez, V., Macas, K., y Ramos, Y. (2021). Educación en tiempos del COVID-19, perspectivadesdelasocioformación. In Revista Dilemas Contemporáneos: Educación, Política y Valores. http://aidipe2015. aidipe.org

Chaves Torres, A. N. (2017). La educación a distancia como respuesta a las necesidades educativas del siglo XXI. Academia y Virtualidad, 10(1), 2341. https://doi.org/10.18359/ravi.2241

Defensoria del Pueblo. (2020). Educacion En Pandemia. https://www.defensoria.gob.pe/ wp-content/uploads/2020/08/Serie-InformesEspeciales-No-027-2020-DP-La-educaciónfrente-a-la-emergencia-sanitaria.pdf

Díaz-Sánchez, C. y Barra-Almagiáb, E. (2017). Resiliencia y satisfacción laboral en profesores de colegios municipales y particulares subvencionados de la comuna de Machalí. Estudios Pedagógicos, 43(1), 75-86.

Dos Santos, M., Ribeiro. S, Scorsolini-Comin2, F., y De Marchi, R. (2021). Ser docente en el contexto de la pandemia de COVID-19: reflexiones sobre la salud mental. Index Enfermería. https://scielo.isciii.es/scielo.php? script=sci_ arttextypid=S1132-12962020000200008

Gómez A, I., y Escobar M, F. (2021). Educación virtual en tiempos de pandemia: Incremento de la desigualdad social en el Perú. Biblioteca Electrónica Científica En Línea, I, 1-13. https:// 
preprints.scielo.org/index.php/scielo/preprint/ view/1996\#.YFvtuoYYVe0.mendeley

Gonzales-Sánchez, A. del C., Mariño, N. R. T., Pelayo, I. L. R., y Ocaña-Fernández, Y. (2021). Management in strengthening social- emotional competence during health emergencies: Gestão no fortalecimento da competência socioemocional durante emergências de saúde. Revista Tempos e Espaços Em Educação, 14(33), e15111. https://doi.org/10.20952/revtee. v14i33.15111

Gordón, F. del R. A. (2020). From face-to-face learning to virtual learning in pandemic times. Estudios Pedagogicos, 46(3), 213-223. https:// doi.org/10.4067/S0718-07052020000300213

López, P., y Gallegos, V. (2014). Prácticas de liderazgo y el rol mediador de la eficacia colectiva en la satisfacción laboral de los docentes. Estudios Pedagógicos (Valdivia), 40(1), 163-178

Minedu. (2020a). Nº 093 RV Aprobar el documento normativo denominado "Orientaciones pedagógicas para el servicio educativo de Educación Básica durante el año 2020 en el marco de la emergencia sanitaria por el Coronavirus COVID-19". https://www.gob.pe/ institucion/minedu/normas-legales/535987093-2020-minedu

Minedu. (2020b). "Orientaciones para el desarrollo del Año Escolar 2020 en las Instituciones Educativas y Programas Educativos de la Educación Básica” (p. 48). file://C:/Users/ coco/Downloads/diego/RVM_N__079-2020MINEDU.pdf

Moreno, E. (2017). Concepciones de práctica pedagógica. Revista Folios, 16, 105. https://doi. org/10.17227/01234870.16folios105.129
Ocaña-Fernández, Y., Valenzuela-Fernández, L., y Morillo-Flores, J. (2020). La competencia digital en el docente universitario. Propósitos y Representaciones, 8(1). https://doi. org/10.20511/pyr2020.v8n1.455

Picón, G., González, G., y Paredes, J. (2020). Desempeño y formación docente en competencias digitales en clases no presenciales durante la pandemia COVID-19. [Performance and Teacher Training in Digital Competences in Non-Presential Classes During the COVID-19 Pandemic]. Universidad Privada María Serrana, Asunción, 1-16. https://preprints.scielo.org/ index.php/scielo/preprint/view/778

Poma, L. S. (2014). Aportes para contextualizar la meritocracia en las políticas y culturas docentes. Revista de Educação PUC-Campinas, 19(2), 111. https://doi.org/10.24220/2318-0870v19n2a2685

Pozos Pérez, K. V., y Tejada Fernández, J. (2018). Competencias Digitales en Docentes de Educación Superior: Niveles de Dominio y Necesidades Formativas. Revista Digital de Investigación En Docencia Universitaria, 12(2), 59-87. https://doi.org/10.19083/ridu.2018.712

Rodríguez-Sosa, J., y Hernández-Sánchez, K. (2018). Problematización de las prácticas docentes y contextualización de la enseñanza. Propósitos y Representaciones, 6(1), 507-524. https://doi. org/10.20511/pyr2018.v6n1.211

Sánchez, M. G., y García, M. D. L. (2017). Satisfacción laboral en los entornos de trabajo. Una exploración cualitativa para su estudio. Scientia et Technica, 22(2), 161-166. http:// www.redalyc.org/pdf/849/84953103007.pdf 
Ventura Montes, U. (2020). Perspectiva del personal docente peruano sobre las razones y las limitaciones que imposibilitan una dedicación continua para con la investigación científica. Revista Educación, 44(2), 219-237.
Zambrano, E. L. (2018). Prácticas pedagógicas para el desarrollo de competencias ciudadanas. Revista Electronica de Investigacion Educativa, 20(1), 69-82. https://doi.org/10.24320/ redie.2018.20.1.1409 